\title{
Competencia Monopolística y Márgenes Bancarios en el Perú: 1995-2005 \\ Gaby Cortez Cortez
}

Resumen : Los resultados encontrados para medir la competencia en el mercado bancario peruano a través del cálculo del $\mathrm{H}$ estadístico de Panzar \& Rosse, sugieren la presencia de un mercado de competencia monopolística, que ha disminuido su nivel de competitividad en los cinco últimos años; lo que de alguna manera ha producido un efecto en el aumento de los márgenes promedio de la banca durante los cuatro últimos años, el que ha sido atenuado por la competencia de los bancos extranjeros.

Palabras claves : Competencia, Hestadístico, margen bancario. 


\section{Introducción}

Los cambios derivados de la reforma financiera de comienzos de los años noventa han producido diversos efectos en el sistema bancario peruano tales como: i) un mayor grado de profundización del sistema bancario, pero que aun se mantiene por debajo del promedio de América Latina, ii) un aumento apreciable del crédito bancario, el que luego de la crisis financiera del 1998-1999 ha disminuido su ritmo de expansión, iii) la eliminación de los topes de las tasas de interés que incentivaron el negocio bancario, pero que todavía mantienen altas tasas en promedio, iv) el aumento significativo de las operaciones en moneda extranjera lo que generó el descalce respectivo, y v) el ingreso de nuevos bancos extranjeros que incrementaron la competencia bancaria; así como la salida apreciable de bancos medianos y pequeños que ha conducido a un mayor grado de concentración y consolidación bancaria, lo cual ha producido efectos en el margen de los bancos.

El trabajo de King y Levine (1993), presenta evidencia empírica que es consistente con el punto de vista de Schumpeter, en el sentido que el sistema financiero puede promover el crecimiento económico, para lo cual emplean datos de 80 países para el período de 1960 a 1989. En dicho trabajo, los autores encuentran que el nivel de desarrollo financiero esta fuertemente asociado con el crecimiento del PBI per cápita, la tasa de acumulación del capital físico y las mejoras en la eficiencia del capital físico. ${ }^{N}$ En la mayor parte de las economías en desarrollo el sistema financiero esta mayormente explicado por el comportamiento del sistema bancario, por lo que su desarrollo es importante para el crecimiento económico de un país, debido a la capacidad que tienen los bancos de asignar recursos financieros hacia las actividades productivas, de consumo y de infraestructura habitacional principalmente. Por tales razones, consideramos que un sistema bancario competitivo y con márgenes adecuados es un elemento importante en el proceso de crecimiento económico del país, por lo que creemos que es necesario estudiar y analizar la relación entre la competencia y los márgenes bancarios durante el período 1995-2005 a través de los ingresos y costos. 


\section{Revisión de la Literatura Empírica sobre Margen Bancario}

El trabajo de Ho y Saunders (1981) sobre los determinantes del margen de interés bancario, hace énfasis en la volatilidad de las tasas de interés altas y su impacto en los márgenes de interés de los bancos o el spread. Este modelo de comportamiento bancario de la hipótesis de cobertura, visualiza al banco en la búsqueda de igualar la maduración de activos y pasivos a fin de evitar el refinanciamiento y considera que el mayor riesgo del portafolio se deriva de las fluctuaciones de las tasas de interés.

Lucia Barrantes (1998) en su estudio sobre el margen financiero del sistema bancario peruano para 1993-1997, considera que los determinantes microeconómicos del margen financiero son la cartera atrasada, la eficiencia de costos operativos, la concentración del mercado, el costo de los recursos, la eficiencia de otros ingresos, la eficiencia por productividad y la rentabilidad de los bancos. Dentro de los factores macroeconómicos se incluyen el grado de monetización, la tasa de encaje marginal, la remuneración al encaje en moneda extranjera, la tasa de inflación, y el riesgo país.

En el trabajo de Demirguc-Kunt y Huizaga (1999), se muestra que las diferencias entre los márgenes de interés y las ganancias bancarias reflejan una variedad de determinantes tales como las características de los bancos, las condiciones macroeconómicas, los impuestos bancarios explícitos e implícitos, las regulaciones sobre seguros de depósitos, la estructura financiera, y los indicadores legales e institucionales. Encuentran que un ratio grande de activos bancarios en relación al PBI y una concentración baja del mercado conducen a márgenes y ganancias más bajas.

El estudio de Barajas (1999) examina los determinantes de los altos márgenes de intermediación observados en el sector bancario de Colombia para más de dos décadas (1974-1996). Se estima una ecuación de la forma reducida en base a un modelo de maximización de la ganancia bancaria que permite una descomposición entre costos operativos, impuestos financieros, poder de mercado, y calidad de préstamos. Se encuentra que a pesar que el margen promedio no cambio entre el período de pre- 
liberalización (1974-1988) y de post liberalización (1991-1996), su composición sí; con un poder de mercado significativamente reducido y un aumento de la calidad de los préstamos.

La investigación de Brock y Rojas (2000) sobre los determinantes de los márgenes bancarios para Argentina, Bolivia, Chile, Colombia, México, Perú y Uruguay durante la mitad de los noventas, encuentra que los altos costos operativos incrementan el margen, así como los niveles altos de préstamos en problemas, a pesar que la magnitud de estos efectos difiere entre países. Adicionalmente a las variables especificas de los bancos, la incertidumbre en el ambiente macroeconómico al que hacen frente los bancos, parece incrementar los márgenes. Los autores hallan que a medida que el margen se amplia, el costo de usar el sistema financiero se vuelve prohibitivo para algunos prestatarios potenciales.

El trabajo de Espino (2006) mide el efecto de la concentración bancaria sobre el margen de las tasas de interés en el sistema bancario del Perú en el periodo 1995-2004, empleando el paradigma de la Estructura-Conducta-Resultado (ECR. El trabajo encuentra que la concentración bancaria tiene un efecto positivo y significativo sobre el margen de las tasas de interés de los bancos. Asimismo, se esboza que las implicancias de política apuntan a que se deba promover la competencia en el sistema bancario con la finalidad de disminuir el efecto de la concentración bancaria sobre el margen de la tasa de interés.

Gastón Gelos (2006) considera que los márgenes de intermediación en América Latina son altos comparados con los estándares internacionales. Su trabajo examina los determinantes de los márgenes de interés de los bancos en esta región usando datos de bancos de 85 países, incluyendo 14 economías Latino Americanas. Los resultados sostienen que América Latina tiene tasas de interés altas, bancos menos eficientes y grandes requerimientos de reservas que en otras regiones, y que estos factores tienen un impacto significativo en los márgenes.

La revisión de la literatura sobre competencia se puede ver en Competencia y 
Eficiencia en el Sector Bancario en el Perú: 1990-2005, que se trabajó en el Instituto de Investigaciones Económicas, ver Cortez, Gaby (2006).

\section{Descripción de la Evidencia sobre el Margen Bancario}

Se define el Margen Bancario o Spread como la diferencia entre la tasa de interés que un banco cobra por los préstamos que otorga y la tasa de interés que paga a sus depositantes. El Spread Ex - ante es la diferencia entre las tasas contractuales cargadas a los préstamos y las tasas pagadas por los depósitos, y el Spread Ex-post es la diferencia entre el ingreso actual por intereses del banco y su gasto actual por intereses para conseguir fondos ${ }^{2}$. En tal sentido, se operativiza la definición de margen bancario de la siguiente manera:

Margen Bancario $=\frac{(\text { Intereses y Comisiones de Cartera Vigente })}{\text { Cartera Vigente }}-\frac{(\text { Intereses de Depósitos })}{\text { Depósitos totales }}$

\subsection{Margen promedio del sistema bancario}

Se encuentra que el Margen promedio del sistema bancario para el período comprendido entre 1995 y 2005 ha experimentado una tendencia a la disminución de 1995 al 2001 - con excepción del año 1999 -, y luego se revierte dicha propensión del 2002 al 2005, al incrementarse dichos márgenes, tal como puede verse en el Gráfico 1. Esta elevación de los márgenes nos estaría indicando cierto retroceso en el proceso de disminución de los mismos, lo que seria una señal de alguna pérdida de competitividad del sistema bancario. Asimismo, se puede observar en este mismo Gráfico que a medida que el número de bancos disminuye en el sistema como resultado de las fusiones, adquisiciones, absorciones, y liquidaciones, el margen bancario se incrementa, especialmente luego que los bancos se consolidan a partir del 2002. 


\subsection{Margen promedio de los bancos grandes}

Se observa que el Margen promedio de los bancos grandes, que comprende a los bancos de Crédito, Continental y Wiese - Sudameris, muestran a lo largo del período de análisis una tendencia a la disminución, con excepción de los años 2003 y 2004, lo que influye de manera importante en la reducción del margen promedio del sistema bancario, tal como puede verse en el Gráfico 2. Contribuye a esta disminución en primer lugar la reducción sostenida del margen del Banco Continental especialmente a partir del año 2000 -, y en segundo lugar al descenso del margen del Banco de Crédito; de tal manera que a partir de ese año, el Banco Continental lidera la reducción del margen bancario en el sistema, ver el Gráfico 3. Por otro lado, si bien es cierto el Banco Wiese - Sudameris tuvo márgenes inferiores al de los otros dos bancos grandes de 1993 al 2001, a partir del año 2002, sus márgenes bancarios se incrementaron de manera significativa, sugiriéndonos su pérdida de competitividad en el sistema.

\section{GRÁFICO 1 \\ MARGEN PROMEDIO DE LA BANCA Y NÚMERO DE BANCOS 1990 - 2005}

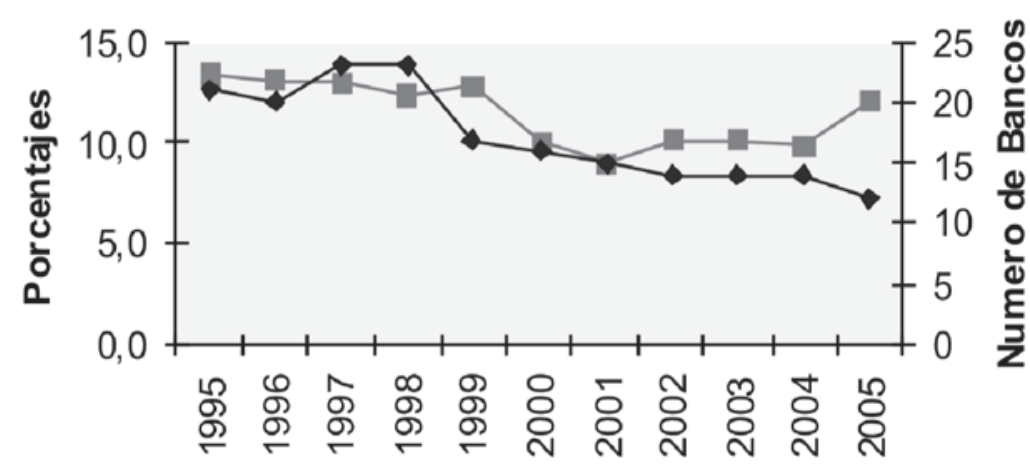

$\rightarrow-$ Promedio de la Banca $\rightarrow-$ Numero de Bancos

Elaboración: Propia 


\subsection{Margen promedio de los bancos medianos}

Los bancos medianos comprenden a los bancos Interbank y Citibank durante todo el período de análisis, y a los bancos de Lima-Sudameris y Bancosur hasta 1998, Latino hasta el 2000, Santander Central Hispano hasta el 2002, y Sudamericano hasta el 2005, siendo estos cinco últimos bancos liquidados, absorbidos o fusionados con otros bancos del sistema.

La Banca Mediana ha tenido márgenes promedio ligeramente superiores a los de la Banca Grande de 1995 al 2000, alcanzando valores inferiores a los de la Banca Grande del 2001 al 2004, contribuyendo de manera especial a la disminución del margen promedio de estos bancos, los Bancos Sudamericano, Santander y Citibank en ese orden. El hecho que los márgenes de la banca mediana están bastante cercanos a los de la banca grande nos estaría indicando que los bancos grandes tienen márgenes altos, ya que estos últimos pueden hacer uso de economías de escala y de extensión, a las que no tienen acceso los bancos medianos. Ver el Gráfico 2 .

GRÁFICO 2

MARGEN DE LA BANCA GRANDE, MEDIANA Y PEQUEÑA 1999 - 2005

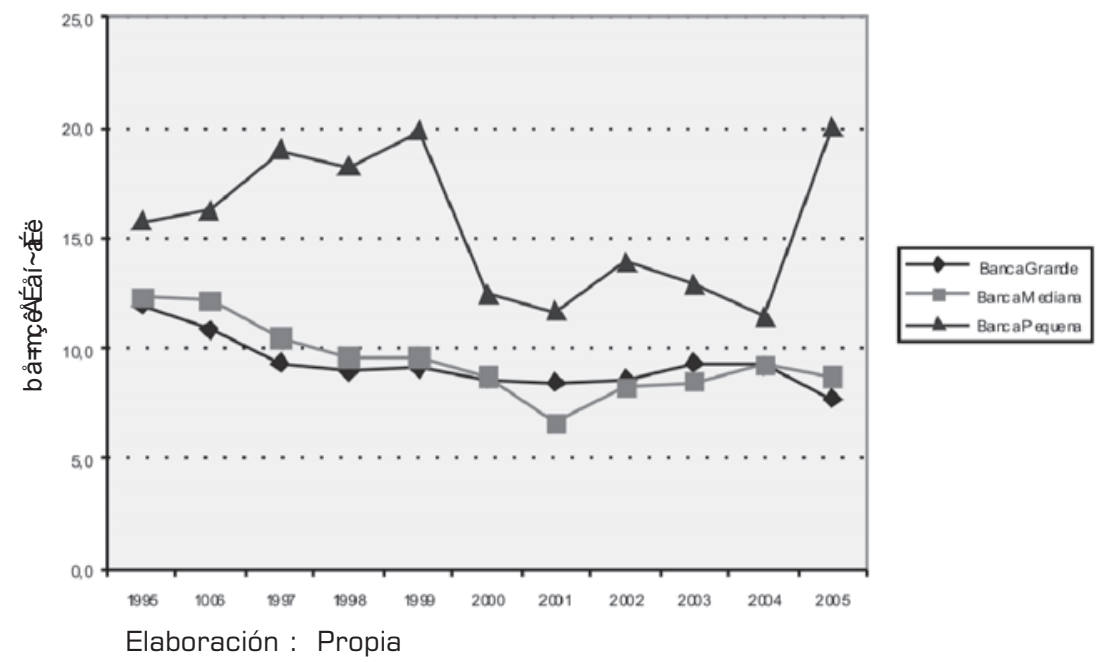




\subsection{Margen promedio de los bancos pequeños}

Los bancos pequeños están constituidos por 21 bancos, de los cuales 5 se han mantenido en el sistema y el resto han sido liquidados, comprados o fusionados con otros bancos. de esta forma son los Bancos Interamericano de Finanzas, Financiero, del Trabajo, MiBanco, y De Comercio, los que en gran medida van a explicar el comportamiento de este grupo de empresas. Los márgenes de la Banca Pequeña son superiores a los de la Banca Mediana y Grande tal como puede verse en el Gráfico 2, indicándonos los altos costos y posibles ineficiencias que estos bancos trasladan al consumidor de los servicios que ofrecen los Bancos Pequeños.

\section{GRÁFICO 3}

MARGEN DE LA BANCA GRANDE : 1995 - 2005

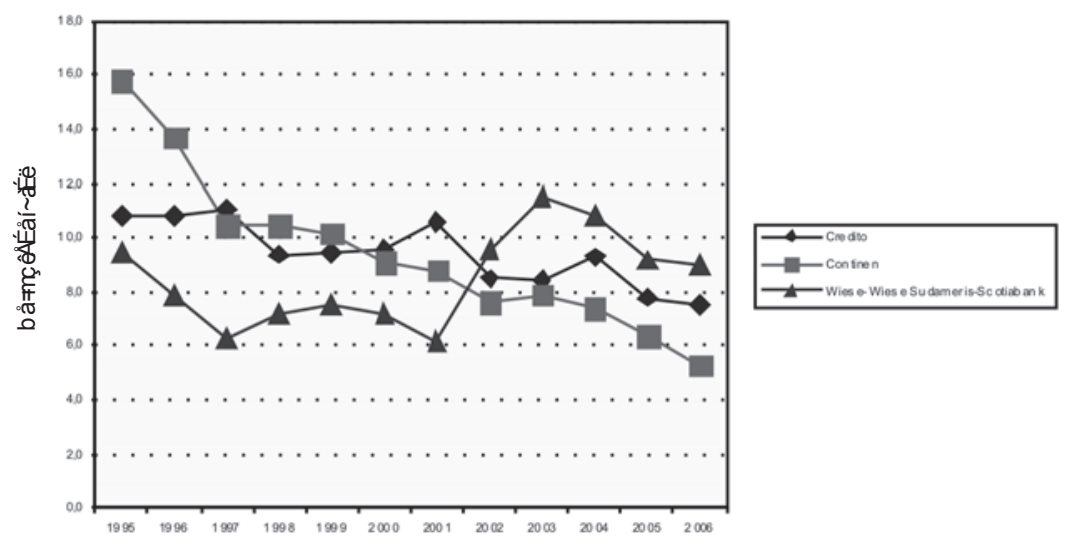

Elaboración : Propia

\section{Estimación Econométrica}

\subsection{La Competencia}

Para medir la competencia en el mercado bancario se emplea el modelo de Panzar \& Rosse (1987), que es una aproximación de la Nueva Organización Industrial Empírica, el que ha sido tomado del trabajo de De Bandt ${ }^{3}$ (2000). El H estadístico de 


\section{Competencia monopolistica y márgenes bancarios en el Perú: 1995-尺005}

Panzar \& Rosse esta definido como la suma de las elasticidades de! ingreso bruto en relación a los costos de los factores, en una ecuación del ingreso de forma reducida. El Hestadístico es una medida que relaciona los cambios producidos en los precios de los inputs (capital, trabajo y costos financieros) sobre los ingresos del banco. Este indice es negativo en el caso de monopolio, positivo pero menor que uno para comperancia monopolística, o igual a uno si se trata de competencia perfecta. La ecuación que empleamos para estimar el valor del H estadístico de Panzar \& Rosse es la siguier:e:

$$
\log R_{i t}=\sum_{j=1}{ }^{j} \alpha_{j} \log W_{i t}{ }^{j}+\sum_{k=1}{ }^{k} \beta_{k} \log S_{i t}{ }^{k}+\sum_{n=1}{ }^{M} Y_{n} X_{i t}{ }^{n}+\varepsilon_{i t}
$$

En donde:

$R_{\text {it }}$ es el ingreso bruto por intereses o ingreso bruto total, $t=1, \ldots$. T en donde $T$ es el número de periodos observados, $i=1, \ldots, \mathrm{I}$, en donde l es ei numero de bancos, $J$ $=3$ inputs, de tal manera que $\mathrm{w}_{\mathrm{it}}$ es un vector tridimensional de precios de factores, $S_{i t}$ es una variable escala que mide el nivel de capacidad a la que el banco opera e incluye al patrimonio y el activo fijo, $\mathrm{X}_{\mathrm{it}}{ }^{\mathrm{n}}$ es un vector de variables exógenas y especificas del banco que pueden cambiar la mezcla de negocios. En este caso, empleamos los depósitos como proporción de los activos y los ingresos no financieros como proporción de los ingresos totales. La ecuación (1) se ha operativizado de la siguiente manera:

$$
R=\beta_{0}+\beta_{1} W A+\beta_{2} W B+\beta_{3} W C+\beta_{4} S A+\beta_{5} S B+\beta_{6} E A+\beta_{7} E B+\beta_{8} E C
$$

Endonde:

$\mathrm{R}=$ Ingreso Financiero, WA $=$ Gastos de personal / Número de empleados, $\mathrm{WB}=$ Intereses pagados $/$ Depósitos + otros pasivos, $\mathrm{WC}=$ Otros gastos / Total de activo, $\mathrm{SA}=$ Patrimonio, $\mathrm{SB}=$ Activo fijo, $\mathrm{EA}=$ Préstamos $/$ Activo Total, $E B=$ Depósitos /Activo, EC = Ingresos no financieros/Ingresos totales

El resultado de la estimación de la ecuación (1) es el siguiente:

$\mathrm{R}=-0.615591+0.132550 \mathrm{WA}+0.384541 \mathrm{WB}-0.072360 \mathrm{WC}+0.599249 \mathrm{SA}+$ $0.409926 \mathrm{SB}+0.003254 \mathrm{EA}-0.003406 \mathrm{~EB}+0.004814 \mathrm{EC}$. 


\section{$\mathrm{R}^{2}=0.933496 \quad$ D.W. $=1.156846 \quad \mathrm{~F}=138.6131$}

Para las regresiones se ha elegido un conjunto de bancos que se han manterido estables antes, durante y después del período de análisis, sin discriminar el hecho de que sean grandes, medianos o pequeños, a fin de obtener una muestra balanceada y representativa.

El H estadístico encontrado es de $0.44473 \mathbf{i}$, el que de acuerdo al poder discriminatorio de Panzar y Rosse ubica a los bancos en un ambiente de competencia monopolística durante el periodo de análisis. $\mathrm{El}^{2}$ es 0.933496 y es bastante alto, indicándonos que el porcentaje de variación de la variable dependiente es explicado por las variables independientes. El F estadístico es de 138.6131, y es una prueba para contrastar la hipótesis nula de que los coeficientes (excepto el intercepto) están cercanos a cero; mientras que el Durbin Watson a pesar de no ser muy alto, para este tipo de regresión de panel es considerado aceptable, debido a que probablemente las operaciones de los bancos pueden influenciar en la de los otros bancos y viceversa.

\subsection{El Margen Bancario}

Para estimar el Margen Bancario se emplea una adaptación del modelo usado por Gastón Gelos ${ }^{4}$ (2006), para evaluar la importancia relativa de distintos factores en la determinación del margen de interés, quedando especificada la ecuación de la siguiente manera:

$$
M I N=\alpha_{0}+\alpha_{1} W A+\alpha_{2} W B+\alpha_{3} W C+\alpha_{4} E M N+\alpha_{5} E M X+\alpha_{6} S A+\alpha_{7} E A
$$

Endonde:

MIN: Margen de interés neto es $=[($ Total de ingresos por intereses $/$ Total de préstamos) - (Total de gastos por intereses / Total de depósitos)] * 100

Competencia: Se mide el grado de competencia en el sistema bancario a través 
Competencia monopolistica y márgenes bancarios en el Perú: 1995-2005

del H de Panzar \& Rosse, y a fin de operativizar de forma anual este factor se ha empleado los tres precios de los input que usan los bancos de la siguiente manera: WA $=$ Gastos de Personal $/$ Depósitos + Préstamos Totales, $\mathbf{W B}=$ Intereses Pagados $/$ Depósitos + Otros pasivos, $\mathbf{W C}=$ Otros Gastos $/$ Total de Activos .

Encaje: Es el costo de los requerimientos de reservas, medido como la tasa de reservas requeridas por los depósitos, tanto en moneda nacional como en moneda extranjera, y se trabaja de la siguiente manera: $\mathbf{E M N}=$ Encaje en moneda nacional, $\mathbf{E M X}=$ Encaje en moneda extranjera

Bancos: que incluye variables específicas de un banco, que en este caso son: SA $=$ Patrimonio, $\mathbf{E A}=$ Préstamos $/$ Activo Total

La estimación de la ecuación (2) nos da el siguiente resultado;

$\mathrm{MIN}=-0.021529 \mathrm{C}+0.472688 \mathrm{WA}-0.088519 \mathrm{WB}+0.300034 \mathrm{WC}+$ $0.250133 \mathrm{EMN}-0.243838 \mathrm{EMX}+0.058215 \mathrm{SA}+0.010274 \mathrm{EA}$
$\mathrm{R}^{2}=0.858967$
D.W. $=0.933095$
$\mathrm{F}=55.68481$

\subsection{Análisis de los Resultados}

El parámetro de los Gastos de Personal / Número de empleados (WA) nos indica que ante una variación de uno por ciento en el ratio de gastos unitarios de personal, el margen de los bancos se incrementara en 0.47 por ciento, es decir, en forma menos que proporcional a dicho gasto, mostrándonos al mismo tiempo la gran significación del costo de estos recursos en el nivel del margen, y sugiriendo la presencia de ciertas ineficiencias en el uso de los gastos de personal de parte de los bancos.

\section{El parámetro de los Intereses pagados / Depósitos + Otros pasivos (WB)} nos indica que ante una variación de uno por ciento de este ratio, el margen bancario 
disminuye en 0.08 por ciento, señalandonos el efecto poco significativo que tiene un aumento de los intereses pagados por los depósitos sobre el margen. Esto probablemente se explique porque las tasas de interés pasivas son tan bajas e irrelevantes, que el efecto intermediación captación de depósitos colocación de recursos, se ve afectado negativamente por el bajo nivel de los intereses pagados por los depósitos. De otro lado, es probable que algunos bancos que tienen facilidad de acceso a recursos externos, hagan uso de ellos para compensar este efecto negativo.

El parámetro de Otros gastos / Total de activos (WC) nos dice que ante una variación de uno por ciento de este ratio, el margen se incrementa en 0.30 por ciento, señalándonos la relevancia que tiene este rubro de gastos en la determinación del margen. Contribuyen a explicar los otros gastos, las prima de seguro de depósitos, gastos diversos, y servicios recibidos de terceros, dentro de los que se incluye el rubro de publicidad y promoción.

El parámetro del Encaje en Moneda Nacional (EMN) y del Encaje en Moneda Extranjera (EMX), nos muestra en el primer caso que ante una variación de uno por ciento del encaje en moneda nacional, el margen se incrementa en 0.25 por ciento, esto debido a que la tasa de encaje promedio para este tipo de moneda durante este período fue bastante baja, alrededor de 7.3 por ciento, lo que le permitió a los bancos tener una mayor capacidad de expansión de sus depósitos; mientras que en el caso del encaje en moneda extranjera, el margen disminuye en 0.24 por ciento, lo que puede ser explicado por la alta tasa de encaje promedio para el período que fue de 36.3 por ciento, lo que ha restringido la capacidad de colocación de recursos de los bancos.

El parámetro del Patrimonio (SA), es positivo y no muy significativo, y nos señala que ante una variación de uno por ciento en el Patrimonio, el margen se incremento en tan solo 0.05 por ciento, mostrándonos que no es importante el volumen de la inversión de los dueños de los bancos en la determinación del margen; pero como es una variable de escala es importante para comparar la magnitud de los bancos dentro del sistema. 


\section{Competencia monopolística y márgenes bancarios en el Perú: 1995-2005}

El parámetro de Préstamos / Activo total (EA), es positivo y no es significativo revelándonos que ante una variación de uno por ciento de este ratio, el margen se incremento en 0.01 por ciento. Este parámetro a captado el efecto negativo de las malas colocaciones de los bancos, lo que a su vez generó préstamos y mora en niveles significativos, así como la disminución del crédito bancario, y la competencia probablemente se ha trasladado a otros rubros, como por ejemplo, el manejo de portafolio de activos, y el manejo de fondos mutuos entre otros.

\section{Conclusión}

Se encuentra que el H estadístico de Panzar \& Rosse para el mercado bancario peruano durante el período 1995-2005 ha sido de 0.444, situándose dentro de los limites correspondientes para clasificarlo como un mercado de competencia monopolística. El margen bancario explicado bajo los términos de la competencia antes indicada, nos muestra que las variables relevantes que determinan la competencia, tienen un efecto significativo en la explicación del margen bancario.

Se encuentra una estrecha relación entre los gastos de personal, así como los otros gastos con el margen bancario, de tal manera que un manejo más eficiente de estos costos de parte de los bancos, puede tener efectos positivos en la disminución del margen.

El margen promedio del sistema bancario ha disminuido de 1995 al 2001, y del 2002 al 2005 se encuentra un aumento del mismo, indicando un retroceso en el comportamiento competitivo de los bancos, generando un menor bienestar para los consumidores finales de los servicios bancarios. 


\section{BIBLIOGRAFÍA}

Barajas, Adolfo., Steiner, Roberto. Interest Spreads in Banking in Colombia, 1974-96. IMF Staff Papers. Volumen 46, No 2, June 1999.

Barrantes, Lucia. Determinantes del margen de intermediación en el caso peruano. Revista Estudios Económicos. BCRP. 2002.

Brock, Phillip L., Rojas Suarez, Liliana. Understanding the behavior of bank spreads in Latin America. Journal of Development Economics. Volume 63 (2000), pages 113-134.

De Bandt, Oliver., Davis, E. Philip. Competition, contestability and market structure in European banking sectors on the eve of EMU. Journal of Banking \& Finance 24 (2000), pages 1045-1066.

Cortez, Gaby. Competencia y Eficiencia en el Sector Bancario en el Perú: 1990-2005. Pensamiento Crítico, Revista del Instituto de Investigaciones Económicas. No 6, Diciembre 2006, paginas 99-114. Facultad de Ciencias Económicas. UNMSM.

Demirguc-Kunt, Ash., Huizinga, Harry. Determinants of Commercial Bank Interest Margins and Profitability: Some International Evidence. The World Bank Economic Review, Volume 13, No 2, pages 379-408. 1999.

Espino, Fredy., Carrera, Cesar. Concentración bancaria y margen de tasas de interés en Perú. Revista Estudios Económicos BCRP. 2006.

Gelos, Gastón. Banking Spreads in Latin America. IMF Working Paper. February 2006.

Ho, Thomas S.Y., Saunders, Anthony. The Determinants of Bank Interest Margins: Theory and Empirical Evidence. Journal of Financial and Quantitative Analysis. Volume XVI, No 4, November 1981.

King, Robert., Levine, Ross. Finance \& Growth: Schumpeter Might Be Right. The Quarterly Journal of Economics. Vol.108, No 3. (August, 1993), pp 717-737. 


\section{Referencias}

1. King y Levine, emplean el trabajo de Joseph Schumpeter (1911), en donde el plantea que los servicios proporcionados por los intermediarios financieros de movilización de ahorros, evaluación de proyectos, manejo de riesgo, monitoreo administrativo, y las facilidades para efectuar transacciones, son esenciales para la innovación tecnológica y el desarrollo económico. Asimismo, Goldsmith (1969) y McKinnon (1973) muestran los lazos cercanos entre el desarrollo económico y financiero.

2. Demirguc-Kunt y Harry Huizaga (1999), sostienen que el spread ex-post es mas útil como medida del margen porque al no incluir los créditos en mora, refleja mejor la situación real de tales activos, por lo cual trabajamos con cartera vigente en lugar de cartera total.

3. De Bandt y Davis (2000), estiman el índice H de Panzar \& Rosse solo para una muestra de bancos en consideración a la falta de información para todos los bancos, así como porque debido a la entrada y salida de algunos bancos dicha información no es completa. En atención a estas razones, también hemos usado una muestra representativa para dicho cálculo.

4. Gelos, Gastón (2006), considera un conjunto de determinantes del margen de los bancos en América Latina, de los cuales se ha escogido aquellos que consideramos son relevantes, y de los que se dispone de información adecuada. 